Pure and Applied Mathematics Quarterly

Volume 7, Number 4

(Special Issue:

In memory of Eckart Viehweg)

$1449-1475,2011$

\title{
Low Degree GW Invariants of Spin Surfaces
}

\author{
Young-Hoon Kiem and Jun Li \\ To the memory of Eckart Viehweg
}

\begin{abstract}
A spin surface $S$ refers to the total space of a line bundle $L$ over a smooth projective curve $D$ satisfying $L^{2}=K_{D}$. A spin surface is canonically equipped with a holomorphic 2-form $\theta$, which gives rise to a cosection $\sigma$ of the obstruction sheaf of the moduli stack $\overline{\mathcal{M}}_{g, n}(S, d)$ of stable maps, thus by [6] the localized GW invariants of $S$. In this paper, we first relate the localized GW invariants of $S$ with the twisted GW invariants of $D$ when certain locally freeness assumption holds. We then analyze in detail a situation in which the aforementioned locally freeness does not hold. Both studies are part of our proof of the Maulik-Pandharipande formulas for low degree GW invariants of surfaces with smooth canonical divisors.
\end{abstract}

Keywords: Localized GW invariant, spin surface, stable map.

\section{INTRODUCTION}

Let $S$ be a smooth surface that is the total space of a theta characteristic of a connected smooth curve $D$, (i.e. it is a line bundle $L$ on $D$ such that $L^{\otimes 2} \cong K_{D}$ ). We call such a surface a spin surface in this paper.

Given a spin surface

$$
p: S \longrightarrow D
$$

Received: Oct. 18, 2010; Revised: Jan. 15, 2011. 
and an integer $d$, we form the moduli $\overline{\mathcal{M}}_{g, n}(S, d)$ of stable morphisms to $S$ of fundamental class $d[D] \in H_{2}(S)$, where $D \subset S$ is the zero section of $p$. Since $L$ is a theta characteristic of $D, \omega_{S}=p^{*} L^{\vee} \otimes p^{*} K_{D} \cong p^{*} L$. Let $\theta \in \Gamma\left(S, \omega_{S}\right)$ be the tautological section of $p^{*} L$, meaning that for any $\left.a \in L\right|_{x}$ where $x \in D, \theta(a)=$ $\left.a \in L\right|_{x}$. Following [6], the holomorphic two-form $\theta$ induces a homomorphism

$$
\sigma: \mathcal{O} b \longrightarrow \mathcal{O}_{\overline{\mathcal{M}}_{g, n}(S, d)}
$$

from the obstruction sheaf $\mathcal{O} b$ of the tautological perfect obstruction theory of $\overline{\mathcal{M}}_{g, n}(S, d)$ to the structure sheaf of $\overline{\mathcal{M}}_{g, n}(S, d)$. Further, this homomorphism is surjective away from $\overline{\mathcal{M}}_{g, n}(D, d) \subset \overline{\mathcal{M}}_{g, n}(S, d)$. Applying the cosection localization of virtual cycles [6], we obtain a localized virtual cycle

$$
\left[\overline{\mathcal{M}}_{g, n}(S, d)\right]_{\text {loc }}^{\mathrm{vir}} \in A_{*} \overline{\mathcal{M}}_{g, n}(D, d) .
$$

This cycle defines the (localized) GW invariants of $S$. Let er $: \overline{\mathcal{M}}_{g, n}(S, d) \rightarrow S^{n}$ be the evaluation morphism, let $\gamma_{1}, \cdots, \gamma_{n} \in H^{*}(S)$, let $\alpha_{1}, \cdots, \alpha_{n} \in \mathbb{Z}^{\geq 0}$, and let $\psi_{i}$ be the first Chern class of the relative cotangent line bundle of the domain curves at the $i$-th marked point. We define the GW invariant of a spin surface $S$ with descendants to be

$$
\left\langle\tau_{\alpha_{1}}\left(\gamma_{1}\right) \cdots \tau_{\alpha_{n}}\left(\gamma_{n}\right)\right\rangle_{g, d}^{S}=\int_{\left[\overline{\mathcal{M}}_{g, n}(S, d)\right]_{\mathrm{loc}}^{\mathrm{vir}}} \tilde{\mathrm{v}}^{*}\left(\gamma_{1} \times \cdots \times \gamma_{n}\right) \cdot \psi_{1}^{\alpha_{1}} \cdots \psi_{n}^{\alpha_{n}} .
$$

The first theorem we prove is

Theorem 1.1. Let $p: S \rightarrow D$ be a spin surface associated to a theta characteristic $L$ of $D$. Let $(\pi, f): \mathcal{C} \rightarrow \overline{\mathcal{M}}_{g, n}(D, d) \times D$ be the universal family. Suppose $R^{1} \pi_{*} f^{*} L$ is locally free, then

$$
\left[\overline{\mathcal{M}}_{g, n}(S, d)\right]_{\text {loc }}^{\mathrm{vir}}=\sum_{\mathcal{W}_{i} \subset \overline{\mathcal{M}}_{g, n}(D, d)}(-1)^{h^{0}\left(f^{*} L\right)}\left[\mathcal{W}_{i}\right]^{\text {vir }} \cap e(V),
$$

where $V$ is the kernel vector bundle of (2.6), the summation is over all connected components $\mathcal{W}_{i}$ of $\mathcal{W}=\overline{\mathcal{M}}_{g, n}(D, d), h^{0}\left(f^{*} L\right)$ is the rank of $\pi_{*} f^{*} L$ over $\mathcal{W}_{i}$, and $e(V)$ is the Euler class of $V$.

This theorem gives the GW invariants of $S$ without insertions. (See Proposition 2.5.) It also proves the degree one part of a conjecture of Maulik-Pandharipande on GW invariants of surfaces, phrased in terms of GW invariants of a spin surface [11, (8)-(9)] (see Theorem 3.2). 
Conjecture 1.2. Let $p: S \rightarrow D$ be a spin surface and $h=g(D)$; let $\gamma=$ $p^{*}[p t]^{P D} \in H^{2}(S)$, where $[p t]^{P D} \in H^{2}(D)$ is the Poincaré dual of the point class in $D$. Then

$$
\begin{gathered}
\left\langle\prod_{i=1}^{n} \tau_{\alpha_{i}}(\gamma)\right\rangle_{[D]}^{S, \bullet}=(-1)^{h^{0}(L)} \prod_{i=1}^{n} \frac{\alpha_{i} !}{\left(2 \alpha_{i}+1\right) !}(-2)^{-\alpha_{i}} ; \\
\left\langle\prod_{i=1}^{n} \tau_{\alpha_{i}}(\gamma)\right\rangle_{2[D]}^{S, \bullet}=(-1)^{h^{0}(L)} 2^{h+n-1} \prod_{i=1}^{n} \frac{\alpha_{i} !}{\left(2 \alpha_{i}+1\right) !}(-2)^{\alpha_{i}} .
\end{gathered}
$$

Here $\bullet$ indicates that the GW invariants are defined for moduli of stable maps with not necessarily connected domains; for definition see Definition 3.1.

Proposition 1.3. The conjecture (1.2) holds.

Even in case $R^{1} \pi_{*} f^{*} L$ is not locally free, by studying the error term we can compare the GW invariants of $S$ with the twisted GW invariants of $D$. This gives the following special case of (1.3).

Proposition 1.4. The conjecture (1.3) holds in case $n=1$.

This Proposition is part of the proof of (1.3) via degeneration developed by the authors. The proof of a degeneration formula of localized GW invariants of spin surfaces will be addressed in a separate paper.

Acknowledgment: The first author is grateful to the Stanford Mathematics department for support and hospitality while he was visiting during the academic year 2005/2006. The second author thanks D. Maulik for sharing with him his computation for an example that is crucial for this paper; he also thanks E. Ionel for stimulating discussions. We thank J. Lee and T. Parker for stimulating questions and for pointing out several oversights in our previous draft.

The second named author is partially supported by NSF grant and the first named author was partially supported by NRF grant 2010-0007786.

\section{GW INVARIANTS AND TWISTED GW INVARIANTS}

We fix a spin surface $p: S \rightarrow D$ that is the total space of a theta characteristic $L$ of a connected, smooth, proper curve $D$ over $\mathbb{C}$ together with its standard 
holomorphic two-form $\theta$ on $S$. We fix a positive $d>0$ and $n, g \in \mathbb{Z}$. For convenience, we abbreviate $\mathcal{M}:=\overline{\mathcal{M}}_{g, n}(S, d)$ and $\mathcal{W}:=\overline{\mathcal{M}}_{g, n}(D, d)$.

The projection $p: S \rightarrow D$ induces a morphism

$$
\bar{p}: \mathcal{M}=\overline{\mathcal{M}}_{g, n}(S, d) \longrightarrow \mathcal{W}=\overline{\mathcal{M}}_{g, n}(D, d) .
$$

At individual map level, in case $u: C \rightarrow S$ is a stable map, then $p \circ u: C \rightarrow D$ is a stable map, and $u$ is determined by a global section in $H^{0}\left(C,(p \circ u)^{*} L\right)$. Conversely, using the embedding $D \subset S$ by the zero section, $\mathcal{W} \subset \mathcal{M}$ is canonically a substack, and fibers of $\bar{p}$ are vector spaces with $\mathcal{W} \subset \mathcal{M}$ its zero section.

Since $D$ is projective, we can pick two vector bundles $E_{1}$ and $E_{2}$ on $\mathcal{W}$ and a sheaf homomorphism

$$
\alpha: E_{1} \longrightarrow E_{2}
$$

(we will not distinguish a vector bundle from its sheaf of sections) so that $\left[E_{1} \rightarrow\right.$ $\left.E_{2}\right]$ is quasi-isomorphic to $\pi_{!} f^{*} L$. By viewing $q: E_{1} \rightarrow \mathcal{W}$ as the total space of $E_{1}$ with $q$ the projection, we can form the pullback bundle $q^{*} E_{2}$ on $E_{1}$ and the associated section $\bar{\alpha} \in \Gamma\left(E_{1}, q^{*} E_{2}\right)$.

Lemma 2.1. The vanishing locus $\bar{\alpha}^{-1}(0)$ is the moduli stack $\mathcal{M}$.

Proof. The proof is straightforward and will be omitted.

We now describe the non-surjective locus of the cosection $\sigma: \mathcal{O} b \rightarrow \mathcal{O}_{\mathcal{M}}$ induced by $\theta$. A stable map $u: C \rightarrow S$ in $\mathcal{M}$ is called $\theta$-null if the composite

$$
u^{*}(\hat{\theta}) \circ d u:\left.\left.T_{C_{\mathrm{reg}}} \longrightarrow u^{*} T_{S}\right|_{C_{\mathrm{reg}}} \longrightarrow u^{*} \Omega_{S}\right|_{C_{\mathrm{reg}}}
$$

vanishes over the regular locus $C_{\text {reg }}$ of $C$. Applying a criterion proved in [6], the locus $\mathcal{M}(\sigma)$ where $\sigma$ fails to be surjective is the collection of all $\theta$-null stable morphisms in $\mathcal{M}$. Since $d>0$, using the explicit form of $\theta$, we see that $\sigma$ is surjective away from $\mathcal{W}=\overline{\mathcal{M}}_{g, n}(D, d) \subset \mathcal{M}$. This shows that the localized virtual cycle satisfies

$$
[\mathcal{M}]_{\mathrm{loc}}^{\mathrm{vir}} \in A_{*} \mathcal{W} .
$$

Using (1.1), we have defined the localized GW invariants $\langle\cdot\rangle_{g, d}^{S}$ of $S$.

We now investigate the relation between the localized GW invariants $\langle\cdot\rangle_{g, d}^{S}$ and the twisted GW invariants of the curve $D$. We let

$$
(\pi, f): \mathcal{C} \longrightarrow \mathcal{W} \times D
$$


be the universal family of $\mathcal{W}=\overline{\mathcal{M}}_{g, n}(D, d)$. The twisted GW invariants of $D$ in this paper refer to the evaluation against $[\mathcal{W}]^{\text {vir }}$ of the usual insertions

$$
\psi_{1}^{\alpha_{1}} \cdots \psi_{n}^{\alpha_{n}} \cdot \operatorname{ev}^{*}(\gamma), \quad \gamma \in H^{*}\left(D^{n}\right)
$$

multiplied (or twisted) by the Chern classes of $\pi_{!} f^{*} L$.

Let

$$
(\tilde{\pi}, \tilde{f}): \tilde{\mathcal{C}} \longrightarrow \mathcal{M} \times S
$$

be the universal family of $\mathcal{M}$. Because $S \rightarrow D$ has affine fibers, the composite $p \circ \tilde{f}: \tilde{\mathcal{C}} \rightarrow D$ is a family of stable morphisms. Therefore, $\tilde{\mathcal{C}}=\mathcal{C} \times \mathcal{W} \mathcal{M}$ and $p \circ \tilde{f}$ is the pullback of $f$.

It was hoped that the localized GW invariants of $S$ can be recovered by the twisted GW invariants of $D$. As was pointed by Maulik, this fails in general. However, in case the sheaf $R^{1} \pi_{*} f^{*} L$ is locally free, this is true up to a sign by Theorem 1.1.

We describe the cosection $\sigma$ in case $R^{1} \pi_{*} f^{*} L$ is locally free.

Lemma 2.2. Let the notation be as above and suppose $d>0$. Assume $R^{1} \pi_{*} f^{*} L$ is locally free. Then $\mathcal{M}$ is the total space of $E_{1}:=\pi_{*} f^{*} L$. Let $\bar{p}: E_{1}=\mathcal{M} \rightarrow \mathcal{W}$ be the projection. Then the homomorphism $\sigma: \mathcal{O b}_{\mathcal{M}} \rightarrow \mathcal{O}_{\mathcal{M}}$ induced by $\theta$ is the composite of $\nu$ and $\bar{\theta}^{\vee}$ :

$$
\mathcal{O} b_{\mathcal{M}} \stackrel{\nu}{\longrightarrow} \bar{p}^{*} E_{1}^{\vee} \stackrel{\bar{\theta}^{\vee}}{\longrightarrow} \mathcal{O}_{\mathcal{M}}
$$

where $\nu$ is surjective and $\bar{\theta}^{\vee}$ is defined by pulling back via $\bar{p}^{*}$ the dual-pairing $E_{1} \otimes E_{1}^{\vee} \rightarrow \mathcal{O}_{\mathcal{W}}$ and using $\mathcal{M}=E_{1}$

Proof. Because the two-form $\theta$ on $S$ is a section of $p^{*} L=\Omega_{S}^{2}$, linear along fibers of $S \rightarrow D$, it induces a section

$$
\bar{\theta} \in H^{0}\left(\mathcal{M}, \tilde{\pi}_{*} \tilde{f}^{*} p^{*} L\right) ;
$$

Since $\theta$ vanishes along $D \subset S$ and is non-degenerate away from $D$, the vanishing locus of its dual

$$
\bar{\theta}^{\vee} \in \operatorname{Hom}\left(R^{1} \tilde{\pi}_{*}\left(\tilde{f}^{*} p^{*} L^{\vee} \otimes \omega_{\tilde{\mathcal{C}} / \mathcal{M}}\right), \mathcal{O}_{\mathcal{M}}\right)
$$

is exactly $\mathcal{W} \subset \mathcal{M}$. 
Since $L \cong L^{\vee} \otimes K_{D}$, we further have a homomorphism

$$
\tilde{f}^{*} p^{*} L \cong \tilde{f}^{*} p^{*} L^{\vee} \otimes \tilde{f}^{*} p^{*} K_{D} \longrightarrow \tilde{f}^{*} p^{*} L^{\vee} \otimes \omega_{\tilde{\mathcal{C}} / \mathcal{M}}
$$

(induced by $\tilde{f}^{*} p^{*} \Omega_{D} \rightarrow \omega_{\tilde{\mathcal{C}} / \mathcal{M}}$ ) that induces a homomorphism between locally free sheaves

$$
R^{1} \tilde{\pi}_{*} \tilde{f}^{*} p^{*} L \longrightarrow R^{1} \tilde{\pi}_{*}\left(\tilde{f}^{*} p^{*} L^{\vee} \otimes \omega_{\tilde{\mathcal{C}} / \mathcal{M}}\right) .
$$

We claim that this homomorphism is surjective. Let $u: C \rightarrow S$ be a positive degree stable map. Since $H^{0}\left(u^{*} p^{*} L\right) \rightarrow H^{0}\left(u^{*} p^{*} L^{\vee} \otimes \omega_{C}\right)$ is injective, its Serre dual $H^{1}\left(u^{*} p^{*} L\right) \rightarrow H^{1}\left(u^{*} p^{*} L^{\vee} \otimes \omega_{C}\right)$ is surjective. The surjectivity of (2.5) follows from the base change property.

Obviously, (2.5) is the pullback via $\bar{p}$ of a similarly defined surjective homomorphism

$$
E_{2}=R^{1} \pi_{*} f^{*} L \longrightarrow R^{1} \pi_{*}\left(f^{*} L^{\vee} \otimes \omega_{\mathcal{C} / \mathcal{W}}\right)=E_{1}^{\vee}
$$

over $\mathcal{W}$.

We claim that the homomorphism $\sigma: \mathcal{O}_{\mathcal{M}} \rightarrow \mathcal{O}_{\mathcal{M}}$ induced by $\theta$ factors through the homomorphism $\bar{\theta}^{\vee}$ in (2.4). Indeed, from the natural morphisms

$$
\tilde{f}^{*}\left(T_{S} \otimes p^{*} L\right)=\tilde{f}^{*}\left(T_{S} \otimes \Omega_{S}^{2}\right) \longrightarrow \tilde{f}^{*} \Omega_{S} \longrightarrow \Omega_{\tilde{\mathcal{C}} / \mathcal{M}} \longrightarrow \omega_{\tilde{\mathcal{C}} / \mathcal{M}}
$$

we obtain a homomorphism $\tilde{f}^{*} T_{S} \rightarrow \tilde{f}^{*} p^{*} L^{\vee} \otimes \omega_{\tilde{\mathcal{C}} / \mathcal{M}}$, and thus a homomorphism

$$
R^{1} \tilde{\pi}_{*} \tilde{f}^{*} T_{S} \longrightarrow R^{1} \tilde{\pi}_{*}\left(\tilde{f}^{*} p^{*} L^{\vee} \otimes \omega_{\tilde{\mathcal{C}} / \mathcal{M}}\right) .
$$

Similar to the proof that the cosection $R^{1} \tilde{\pi}_{*} \tilde{f}^{*} T_{S} \rightarrow \mathcal{O}_{\mathcal{M}}$ lifts to $\mathcal{O} b_{\mathcal{M}} \rightarrow \mathcal{O}_{\mathcal{M}}$ ([6, Lemma 6.1]), the composition of (2.7) with the natural homomorphism

$$
\gamma: \mathcal{E} x t_{\tilde{\pi}}^{1}\left(\Omega_{\tilde{\mathcal{C}} / \mathcal{M}}, \mathcal{O}_{\tilde{\mathcal{C}}}\right) \longrightarrow R^{1} \tilde{\pi}_{*} \tilde{f}^{*} T_{S}
$$

is zero. Using $\mathcal{O} b_{\mathcal{M}}=\operatorname{coker}\{\gamma\}$, (2.7) lifts to a homomorphism

$$
\nu: \mathcal{O} b_{\mathcal{M}} \longrightarrow R^{1} \tilde{\pi}_{*}\left(\tilde{f}^{*} p^{*} L^{\vee} \otimes \omega_{\tilde{\mathcal{C}} / \mathcal{M}}\right)=\bar{p}^{*} E_{1}^{\vee},
$$

which by construction satisfies $\bar{\theta}^{\vee} \circ \nu=\sigma$. This proves (2.3).

Because $R^{1} \tilde{\pi}_{*} \tilde{f}^{*} p^{*} L \rightarrow \mathcal{O} b_{\mathcal{M}}$ composed with (2.8) is (2.5), and because (2.5) is surjective, $\nu$ is surjective. Since $R^{1} \tilde{\pi}_{*}\left(\tilde{f}^{*} p^{*} L^{\vee} \otimes \omega_{\tilde{\mathcal{C}} / \mathcal{M}}\right)$ is dual to $\tilde{\pi}_{*} \tilde{f}^{*} p^{*} L=$ $\bar{p}^{*} \pi_{*} f^{*} L=\bar{p}^{*} E_{1}$ and since $\mathcal{M}$ is the total space of $E_{1}$, one checks that $\bar{\theta}^{\vee}$ is obtained by pulling back the dual pairing $E_{1}^{\vee} \otimes E_{1} \rightarrow \mathcal{O}_{\mathcal{W}}$ and using $\mathcal{M}=E_{1}$. This proves the Lemma. 
We now prove Theorem 1.1.

Proof of Theorem 1.1. We pick a vector bundle (locally free sheaf) $F$ on $\mathcal{M}$ and a surjective $F \rightarrow \mathcal{O b}_{\mathcal{M}}$. Since $S$ is quasi-projective, such $F$ exists. The obstruction theory of $\mathcal{M}$ provides us a cone cycle $C_{\mathcal{M}} \subset F$, which is the pullback of the intrinsic normal cone $[2,10]$. We let $\bar{\sigma}: F \rightarrow \mathcal{O}_{\mathcal{M}}$ be the composite of $F \rightarrow \mathcal{O} b_{\mathcal{M}}$ with $\sigma$. By the definition of localized virtual cycles [6],

$$
[\mathcal{M}]_{\mathrm{loc}}^{\mathrm{vir}}=s_{F, \bar{\sigma}}^{!}\left(\left[C_{\mathcal{M}}\right]\right) \in A_{*} \mathcal{W}
$$

where $s_{F, \bar{\sigma}}: Z_{*} \mathcal{M}(\sigma) \rightarrow A_{*} \mathcal{W}$ is the localized Gysin map constructed in [6]. Here $\mathcal{M}(\sigma)=\mathcal{W} \cup \operatorname{ker}\left\{\left.\bar{\sigma}\right|_{\mathcal{M}-\mathcal{W}}\right\}$.

Let $F_{0}=\left.F\right|_{\mathcal{W}}$ and let $C_{0}=C_{\mathcal{M}} \times_{\mathcal{M}} \mathcal{W} \subset F_{0}$; pulling back via $\bar{p}$, we obtain a pair

$$
C:=\bar{p}^{*} C_{0} \subset \bar{p}^{*} F_{0} .
$$

We claim that we can find a homomorphism $\bar{\sigma}^{\prime}: \bar{p}^{*} F_{0} \rightarrow \mathcal{O}_{\mathcal{M}}$ such that

$$
[\mathcal{M}]_{\mathrm{loc}}^{\mathrm{vir}}=s_{F, \bar{\sigma}}^{!}\left(\left[C_{\mathcal{M}}\right]\right)=s_{\bar{p}^{*} F_{0}, \bar{\sigma}^{\prime}}([C]) \in A_{*} \mathcal{W} .
$$

Let $t \in \mathbf{A}^{1} \backslash 0$ and let $\zeta_{t}: S \rightarrow S$ be the scaling of the fibers of $S \rightarrow D$ by $t$, i.e. $\zeta_{t}(v)=t v$. The morphism $\zeta_{t}: S \rightarrow S$ induces a morphism $\bar{\zeta}_{t}: \mathcal{M}=$ $\overline{\mathcal{M}}_{g, n}(S, d) \rightarrow \mathcal{M}$ that maps $[u] \in \mathcal{M}$ to $\left[\zeta_{t} \circ u\right] \in \mathcal{M}$. Clearly, it is also the scaling of the fibers of $\mathcal{M}=E_{1} \rightarrow \mathcal{W}$ by $t$.

We now form the pullback sequence

$$
\bar{\zeta}_{t}^{*} C_{\mathcal{M}} \subset \bar{\zeta}_{t}^{*} F \longrightarrow \bar{\zeta}_{t}^{*} \mathcal{O} b_{\mathcal{M}} \stackrel{\bar{\zeta}_{t}^{*} \nu}{\longrightarrow} \bar{p}^{*} E_{1}^{\vee} \stackrel{t^{-1} \bar{\zeta}_{t}^{*} \bar{\theta}^{\vee}}{\longrightarrow} \mathcal{O}_{\mathcal{M}}
$$

Here since $p \circ \zeta_{t}=p$, we have that $\bar{p} \circ \bar{\zeta}_{t}=\bar{p}$; thus we can replace $\bar{\zeta}_{t}^{*} \bar{p}^{*} E_{1}^{\vee}$ by $\bar{p}^{*} E_{1}^{\vee}$. When $t$ specializes to $0 \in \mathbf{A}^{1}, \bar{\zeta}_{t}^{*} F$ specializes to $\bar{p}^{*} F_{0}$. Denoting by $\tilde{\nu}_{0}: F_{0} \rightarrow E_{1}^{\vee}$ the restriction to $\mathcal{W}$ of the composite of $F \rightarrow \mathcal{O} b_{\mathcal{M}}$ with $\nu$, then the composite of the first two arrows (in the sequence) specializes to $\bar{p}^{*} \tilde{\nu}_{0}$. By direct checking, for all $t \neq 0, t^{-1} \bar{\zeta}_{t}^{*} \bar{\theta}^{\vee}=\bar{\theta}^{\vee}$. Finally, since $\mathcal{M} \rightarrow \mathcal{W}$ is the total space of the vector bundle $E_{1} \rightarrow \mathcal{W}$, for any closed $u \in \mathcal{W}$, the restriction $C_{\mathcal{M}} \times{ }_{\mathcal{M}} \bar{p}^{-1}(u)$ is flat over $\bar{p}^{-1}(u)$. Thus the cones $\bar{\zeta}_{t}^{*} C_{\mathcal{M}}$ specializes to $C:=\bar{p}^{*} C_{0}$. (Recall $C_{0}=C_{\mathcal{M}} \times \mathcal{M} \mathcal{W}$.)

In conclusion, the sequence (2.10) specializes to

$$
C \subset \bar{p}^{*} F_{0} \stackrel{\bar{p}^{*} \tilde{\nu}_{0}}{\longrightarrow} \bar{p}^{*} E_{1}^{\vee} \stackrel{\bar{\theta}^{\vee}}{\longrightarrow} \mathcal{O}_{\mathcal{M}} .
$$


Let $\bar{\sigma}^{\prime}: \bar{p}^{*} F_{0} \rightarrow \mathcal{O}_{\mathcal{M}}$ be the composition of the two arrows above. Since the support of $C_{\mathcal{M}}$ lies in the kernel of $F \rightarrow \mathcal{O}_{\mathcal{M}}$, the support of $C$ lies in the kernel of $\bar{\sigma}^{\prime}$. Finally, because the localized Gysin map preserves rational equivalence [6], and because $t \in \mathbf{A}^{1}, s_{F, \bar{\sigma}}^{!}\left(\left[C_{\mathcal{M}}\right]\right)$ is rationally equivalent to $s_{\bar{p}^{*} F_{0}, \bar{\sigma}^{\prime}}^{!}([C])$. This proves $(2.9)$.

We now investigate the cone $C_{0} \subset F_{0}$. Since $\mathcal{W}=\overline{\mathcal{M}}_{g, n}(D, d)$, and since $\left.T_{S}\right|_{D} \cong T_{D} \oplus L$, for $(\pi, f): \mathcal{C} \rightarrow \mathcal{W} \times D$ the universal family of $\mathcal{W}$,

$$
R^{1} \pi_{*} f^{*} T_{S} \cong R^{1} \pi_{*} f^{*} T_{D} \oplus R^{1} \pi_{*} f^{*} L .
$$

Further, since $f(\mathcal{C}) \subset D$, the homomorphism $\mathcal{E} x t_{\pi}^{1}\left(\Omega_{\mathcal{C} / \mathcal{W}}, \mathcal{O}_{\mathcal{C}}\right) \rightarrow R^{1} \pi_{*} f^{*} T_{S}$ factors through $R^{1} \pi_{*} f^{*} T_{D} \subset R^{1} \pi_{*} f^{*} T_{S}$. Because

$$
\mathcal{O} b_{\mathcal{W}}=\operatorname{coker}\left\{\mathcal{E} x t_{\pi}^{1}\left(\Omega_{\mathcal{C} / \mathcal{W}}, \mathcal{O}_{\mathcal{C}}\right) \rightarrow R^{1} \pi_{*} f^{*} T_{D}\right\}
$$

and

$$
\left.\mathcal{O} b_{\mathcal{M}}\right|_{\mathcal{W}}=\operatorname{coker}\left\{\mathcal{E} x t_{\pi}^{1}\left(\Omega_{\mathcal{C} / \mathcal{W}}, \mathcal{O}_{\mathcal{C}}\right) \rightarrow R^{1} \pi_{*} f^{*} T_{S}\right\}
$$

we have

$$
\left.\mathcal{O} b_{\mathcal{M}}\right|_{\mathcal{W}} \cong \mathcal{O} b_{\mathcal{W}} \oplus R^{1} \pi_{*} f^{*} L=\mathcal{O} b_{\mathcal{W}} \oplus E_{2}
$$

By tracing through the construction of the homomorphism $\nu$ in (2.8), we see that $\left.\nu\right|_{\mathcal{W}}:\left.\mathcal{O} b_{\mathcal{M}}\right|_{\mathcal{W}} \rightarrow E_{1}^{\vee}$ factors through

$$
\left.\mathcal{O} b_{\mathcal{M}}\right|_{\mathcal{W}}=\mathcal{O} b_{\mathcal{W}} \oplus E_{2} \stackrel{\mathrm{pr}}{\longrightarrow} E_{2} \stackrel{(2.6)}{\longrightarrow} E_{1}^{\vee}=R^{1} \pi_{*}\left(f^{*} L^{\vee} \otimes \omega_{\mathcal{C}} / \mathcal{W}\right) .
$$

Let $\left.F_{0} \rightarrow \mathcal{O} b_{\mathcal{M}}\right|_{\mathcal{W}}=\mathcal{O} b_{\mathcal{W}} \oplus E_{2}$ be the restriction of $F \rightarrow \mathcal{O} b_{\mathcal{M}}$ to $\mathcal{W}$, and let $W_{0} \subset F_{0}$ be the kernel bundle of the induced $F_{0} \rightarrow E_{2}$. Thus $W_{0} \rightarrow \mathcal{O} b_{\mathcal{W}}$ is a quotient homomorphism. Because $\mathcal{M}$ is a vector bundle over $\mathcal{W}$, it is direct to check that $C_{0} \subset F_{0}$ lifts to $C_{0} \subset W_{0}$ and is indeed the pullback of the virtual normal cone of the obstruction theory of $\mathcal{W}$ via $W_{0} \rightarrow \mathcal{O} b_{\mathcal{W}}$. In particular, $[\mathcal{W}]^{\text {vir }}=s_{W_{0}}^{!}\left(\left[C_{0}\right]\right)$.

We now prove Theorem 1.1. Following the basic construction of the localized Gysin map [6], we first blow up $\mathcal{M}$ along $\mathcal{W}$. Since $\mathcal{M}=E_{1}$ is a vector bundle over $\mathcal{W}$, the blow-up $\tilde{\mathcal{M}}$ is an $\tilde{\mathbb{A}}^{r_{1}}$-bundle over $\mathcal{W}$, where $\tilde{\mathbb{A}}^{r_{1}}$ is the blow-up of $\mathbb{A}^{r_{1}}$ at its origin and $r_{1}=\operatorname{rank} E_{1}$. We let $\rho: \tilde{\mathcal{M}} \rightarrow \mathcal{M}$ be the blow-up morphism; let $q=\bar{p} \circ \rho: \tilde{\mathcal{M}} \rightarrow \mathcal{W}$, and let $\tilde{C}=q^{*} C_{0} \subset q^{*} F_{0}$ be the proper transform of $\bar{p}^{*} C_{0} \subset \bar{p}^{*} F_{0}$. 
Let $\mathcal{E} \subset \tilde{\mathcal{M}}$ be the exceptional divisor of $\rho$, which is a $\mathbb{P}^{r_{1}-1}$-bundle over $\mathcal{W}$. The pullback $\rho^{*} \bar{\sigma}^{\prime}$ lifts to a surjective homomorphism $q^{*} F_{0} \rightarrow \mathcal{O}_{\tilde{\mathcal{M}}}(-\mathcal{E})$. Let $\tilde{G}=\operatorname{ker}\left\{q^{*} F_{0} \rightarrow \mathcal{O}_{\tilde{\mathcal{M}}}(-\mathcal{E})\right\}$. By the definition of $\tilde{\mathcal{C}}$ and [6], the support of $\tilde{C}$ lies in $\tilde{G}$. Further, since $W_{0} \subset F_{0}$ is the kernel subbundle of $F_{0} \rightarrow E_{2}, q^{*} W_{0}$ is a subbundle of $\tilde{G}$ that contains the support of $C$.

We let $\rho(\sigma): \mathcal{E} \rightarrow \mathcal{W}$ denote the projection. For the moment, we assume $\mathcal{W}$ is connected. By the definition of localized Gysin map,

$s_{q^{*} F_{0}, \bar{\sigma}^{\prime}}^{\prime}([C])=\rho(\sigma)_{*}\left([-\mathcal{E}] \cdot s_{\tilde{G}}^{!}([\tilde{C}])\right)=\rho(\sigma)_{*}\left([-\mathcal{E}] \cdot c_{r_{2}-1}\left(\tilde{G} / q^{*} W_{0}\right) \cdot s_{q^{*} W_{0}}^{!}([\tilde{C}])\right)$.

Here in the expression, $s_{\tilde{G}}^{!}$is the Gysin map of intersecting the zero section of $\tilde{G}$, $[\mathcal{E}]: A_{*} \tilde{\mathcal{M}} \rightarrow A_{*} \mathcal{E}$ is intersecting with the Cartier divisor $\mathcal{E}$ and $r_{2}=\operatorname{rank} E_{2}$.

By the projection formula,

$$
s_{q^{*} W_{0}}^{!}([\tilde{C}])=s_{q^{*} W_{0}}^{!}\left(\left[q^{*} C_{0}\right]\right)=q^{*} s_{W_{0}}^{!}\left(\left[C_{0}\right]\right)=q^{*}[\mathcal{W}]^{\text {vir }} .
$$

As to the quotient $\tilde{G} / q^{*} W_{0} \cong q^{*} E_{2} / \mathcal{O}_{\tilde{M}}(-\mathcal{E})$, since $\bar{p}^{*} E_{2} \rightarrow \mathcal{O}_{\mathcal{M}}$ factors through $\bar{p}^{*} E_{2} \rightarrow \bar{p}^{*} E_{1}^{\vee}$ and since $r_{2}-1$ is the rank of $q^{*} E_{2} / \mathcal{O}_{\tilde{M}}(-\mathcal{E})$, by letting $V$ be the kernel of $E_{2} \rightarrow E_{1}^{\vee}$, we have

$$
c_{r_{2}-1}\left(\tilde{G} / q^{*} W_{0}\right)=c_{r_{2}-1}\left(q^{*} E_{2} / \mathcal{O}_{\tilde{\mathcal{M}}}(-\mathcal{E})\right)=c_{r_{2}-r_{1}}\left(q^{*} V\right) \cdot c_{r_{1}-1}\left(q^{*} E_{1}^{\vee} / \mathcal{O}_{\tilde{\mathcal{M}}}(-\mathcal{E})\right) \text {. }
$$

This proves

$$
\begin{aligned}
\rho(\sigma)_{*}\left([-\mathcal{E}] \cdot c_{r_{2}-1}\left(\tilde{G} / q^{*} W_{0}\right) \cdot s_{q^{*} W_{0}}^{!}([\tilde{C}])\right. & =\rho(\sigma)_{*}\left(-[\mathcal{E}]^{r_{1}} \cdot q^{*}\left(c_{r_{2}-r_{1}}(V)[\mathcal{W}]^{\text {vir }}\right)\right) \\
& =(-1)^{r_{1}} c_{r_{2}-r_{1}}(V)[\mathcal{W}]^{\text {vir }} .
\end{aligned}
$$

Here since $\mathcal{E} \subset \tilde{\mathcal{M}}$ is the exceptional divisor of the blowup of the zero section of the rank $r_{1}$ bundle $E_{1}$ over $\mathcal{W},[\mathcal{E}]^{r_{1}}=(-1)^{r_{1}-1}[\mathcal{W}]$. This proves Theorem $1.1 \mathrm{in}$ case $\mathcal{W}$ is connected.

When $\mathcal{W}$ is not connected, we can perform the same argument to each of its connected components, and derive the stated formula in Theorem 1.1. This completes the proof.

Remark 2.3. The sign $(-1)^{r}$ in the formula can be seen by the following toy case. Take the scheme $M=\mathbf{A}^{r}$ with obstruction sheaf $E=\mathcal{O}_{M}^{\oplus r}$. Since $M$ is smooth, its virtual normal cone is the zero section $0_{E} \subset E$. The usual Gysin map gives $s_{E}^{!}\left[0_{E}\right]=0 \in A_{0} M$. In case we are given a cosection $\sigma: E \rightarrow \mathcal{O}_{M}$, say given by a non-degenerate bilinear form on $\mathbb{C}^{r}$. Then $\sigma$ is surjective away from $0 \in M$, and $s_{E, \sigma}\left[0_{E}\right]=(-1)^{r} \in A_{0}\{0\}$. 
Topologically, this number can be computed by picking a continuous section u of $E$ such that (1) the graph $\Gamma_{u}$ of $u$ is transversal to $0_{E}$; (2) away from a unit ball in $M$, we have $\sigma \circ u=1$. Then $\operatorname{deg} s_{E, \sigma}^{!}\left[0_{E}\right]$ is the degree of the intersection $\Gamma_{u} \cap 0_{E}$. In the case presented, we get $(-1)^{r}$.

Corollary 2.4. Suppose furthermore that $\pi_{*} f^{*} L$ is a trivial vector bundle on $\mathcal{W}$, and let $l$ be the rank of $-\pi_{!} f^{*} L=R^{1} \pi_{*} f^{*} L-\pi_{*} f^{*} L$, then

$$
[\mathcal{M}]_{\mathrm{loc}}^{\mathrm{vir}}=(-1)^{h^{0}\left(f^{*} L\right)}[\mathcal{W}]^{\mathrm{vir}} \cap c_{l}\left(-\pi_{!} f^{*} L\right) .
$$

Proof. Observe that $e(V)=c_{l}\left(-\pi_{!} f^{*} L\right)$ under the assumptions.

Proposition $2.5([9])$. Let $S$ be the total space of a theta characteristic $L$ of a genus $h$ smooth curve $D$; let $g=d(g(D)-1)+1$. Then

$$
\langle 1\rangle_{d, g}^{S}=\sum_{u: d \text {-fold étale cover of } D} \frac{(-1)^{h^{0}\left(u^{*} L\right)}}{|\operatorname{Aut}(u)|} .
$$

Proof. This choice of $g$ forces any $u \in \overline{\mathcal{M}}_{g}(D, d)$ to be a $d$-fold étale cover of $D$. Since an étale cover $u: C \rightarrow D$ is a smooth isolated point in $\overline{\mathcal{M}}_{g}(D, d)$, a direct application of Corollary 2.4 gives the stated formula.

This Proposition was proved by Lee-Parker [9] using analytic methods.

\section{LOW-DEGREE GW INVARIANTS}

In this section, we study Maulik-Pandharipande's conjecture on low degree GW invariants of a spin surface for possibly disconnected domains.

Definition 3.1. For integers $n, \chi$ and $d>0$, we let $\overline{\mathcal{M}}_{\chi, n}(S, d) \bullet$ be the moduli of stable morphisms $u: C \rightarrow S$ from not necessarily connected n-pointed nodal curves $C$ for which $\chi\left(\mathcal{O}_{C}\right)=\chi$ and $u_{*}([C])=d[D]$, and such that the restriction of $u$ to each connected component of $C$ is non-constant.

As usual, we call $u$ stable when the automorphism group of $u$ is finite. The moduli space $\overline{\mathcal{M}}_{\chi, n}(S, d)$ is étale covered by disjoint union of products

$$
\Phi: \coprod_{\alpha \in \Lambda} \prod_{i=1}^{k_{\alpha}} \overline{\mathcal{M}}_{g_{i}, n_{i}}\left(S, d_{i}\right) \longrightarrow \overline{\mathcal{M}}_{\chi, n}(S, d)^{\bullet},
$$


where $\Lambda \ni \alpha=\left(g_{i}, n_{i}, d_{i}\right)_{i=1}^{k_{\alpha}}$ runs over all partitions $d_{1}+\cdots+d_{k_{\alpha}}=d$ with $d_{i}>0$, partitions $\mathbf{n}_{1} \cup \cdots \cup \mathbf{n}_{k_{\alpha}}=\{1, \cdots, n\}$ with $\left|\mathbf{n}_{i}\right|=n_{i}$, and $k_{\alpha}-\sum g_{i}=\chi$.

We now use the holomorphic two-form $\theta$. The preceding discussion of localized GW invariants can be adopted to define localized virtual cycle $\left[\overline{\mathcal{M}}_{g_{i}, n_{i}}\left(S, d_{i}\right)\right]_{\text {loc }}^{\mathrm{vir}}$. We define

$$
\left[\overline{\mathcal{M}}_{\chi, n}(S, d)^{\bullet}\right]_{\mathrm{loc}}^{\mathrm{vir}}=\Phi_{*}\left(\sum_{\alpha \in \Lambda} \frac{1}{|\operatorname{Aut} \alpha|} \cdot \prod_{i=1}^{k_{\alpha}}\left[\overline{\mathcal{M}}_{g_{i}, \mathbf{n}_{i}}\left(S, d_{i}\right)\right]_{\mathrm{loc}}^{\mathrm{vir}}\right),
$$

where Aut $\alpha$ is the automorphism group of $\alpha=\left(d_{i}, \mathbf{n}_{i}, g_{i}\right)_{i=1}^{k_{\alpha}}$.

Consequently, we define the localized GW invariants, of stable maps with not necessarily connected domains but with non-constant restrictions to any connected components, to be

$$
\left\langle\tau_{\alpha_{1}}\left(\gamma_{1}\right) \cdots \tau_{\alpha_{n}}\left(\gamma_{n}\right)\right\rangle_{\chi, d[D]}^{S, \bullet}, \quad \gamma_{i} \in H^{*}(D, \mathbb{Z}) .
$$

(Here we will not include the subscript "loc" into the notation since $S$ is a spin surface and only localized GW invariants make sense.)

Following the convention, since (3.1) is possibly non-trivial only when

$$
-\chi=d(h-1)+\sum_{i=1}^{n} \alpha_{i}, \quad \alpha_{i} \in \mathbb{Z}_{\geq 0}, \quad h=g(D),
$$

we shall omit the reference to $\chi$ in the notation of (3.1) with the understanding that it is given by (3.2).

This section is devoted to a study of Conjecture 1.2 of Maulik and Pandharipande. The first identity follows directly from Corollary 2.4.

Theorem 3.2. Let the condition be as in Conjecture 1.2. Then the identity (1.2) holds.

Proof. Let $-\chi=h-1+\sum \alpha_{i}$, and denote

$$
M=\overline{\mathcal{M}}_{\chi, n}(S, 1)^{\bullet} \quad \text { and } \quad W=\overline{\mathcal{M}}_{\chi, n}(D, 1)^{\bullet} .
$$

Since maps in $M$ are of degree one, their domains must be connected. Likewise the same holds for $W$.

Let $(\pi, f): \mathcal{C} \rightarrow W \times D$ be the universal family. Because maps in $W$ have degree one, a direct check shows that $\pi_{*} f^{*} L \cong H^{0}(L) \otimes \mathcal{O}_{W}$ and satisfies the base change property. Hence $R^{1} \pi_{*} f^{*} L$ is locally free and $\pi_{*} f^{*} L$ is a trivial bundle. 
Applying Corollary 2.4, we conclude that

$$
\left\langle\prod_{i=1}^{n} \tau_{\alpha_{i}}(\gamma)\right\rangle_{[D]}^{S, \bullet}=(-1)^{h^{0}(L)} \int_{[W]^{\mathrm{vir}}} \prod \psi_{i}^{\alpha_{i}} e v_{i}^{*}(\gamma) \cap c_{t o p}\left(-\pi ! f^{*} L\right)
$$

is a twisted GW invariant of $D$ with sign $(-1)^{h^{0}(L)}$. From [3], we can readily evaluate them and obtain (1.2).

The degree two case follows from the combination of a degeneration formula and the special case $n=1$. Since the proof of a degeneration formula requires an extensive study of obstruction theory of the family, we will prove it in a separate paper [7]. In this section, we prove a special case of (1.3) that will be part of the proof of (1.3) using degeneration.

Proposition 3.3 ( $h=0$ case). Let $S$ be the total space of $\mathcal{O}_{\mathbb{P}^{1}}(-1)$. Then

$$
\left\langle\prod_{i=1}^{n} \tau_{\alpha_{i}}(\gamma)\right\rangle_{2\left[\mathbb{P}^{1}\right]}^{S, \bullet}=2^{n-1} \prod_{i=1}^{n} \frac{\alpha_{i} !}{\left(2 \alpha_{i}+1\right) !}(-2)^{\alpha_{i}}
$$

Proof. Since $\mathbb{P}^{1} \subset \mathcal{O}_{\mathbb{P}^{1}}(-1)$ is rigid, the moduli space of stable maps to $\mathcal{O}_{\mathbb{P}^{1}}(-1)$ is proper and the localized GW invariant coincides with the twisted GW invariant of $\mathbb{P}^{1}$ by Corollary 2.4. Hence, (3.4) follows from the differential equations for the twisted invariants in [3].

The remaining part of this paper is devoted to a proof of

Proposition 3.4. Let $p: S \rightarrow D$ be an arbitrary spin surface and $h=g(D) \geq 1$. Then (1.3) holds for $n=1$. Namely,

$$
\left\langle\tau_{1}(\gamma)\right\rangle_{2[D]}^{S, \bullet}=(-1)^{h^{0}(L)}\left(\frac{2^{h}}{-3}\right)
$$

We first describe the moduli space $\overline{\mathcal{M}}_{\chi, 1}(D, 2)^{\bullet}$. Let $\nu: \Sigma_{\nu} \rightarrow D$ be a degree two étale morphism ( $\Sigma_{\nu}$ can possibly be disconnected). Picking a point $p \in \Sigma_{\nu}$, attaching an elliptic curve $E$ to $p$ and requiring $u: \Sigma_{\nu} \cup E \rightarrow D$ be $\nu$ on $\Sigma_{\nu}$ and be constant on $E,[u] \in \overline{\mathcal{M}}_{\chi}(D, 2)^{\bullet}$. Since $\nu$ has a $\mathbb{Z}_{2}$-symmetry, attaching the same $E$ to $p$ or to the other point in $\nu^{-1}(\nu(p))$ gives the same stable map. This shows that each $\nu$ gives an irreducible component of $\overline{\mathcal{M}}_{\chi}(D, 2)^{\bullet}$ that is isomorphic to $D \times \overline{\mathcal{M}}_{1,1}$. An easy check shows that this is a connected component of $\overline{\mathcal{M}}_{\chi}(D, 2)^{\bullet}$ 
and this component is smooth. We denote this component and its preimage under the forgetful morphism $\varphi: \overline{\mathcal{M}}_{\chi, 1}(D, 2)^{\bullet} \rightarrow \overline{\mathcal{M}}_{\chi}(D, 2)^{\bullet}$ by

$$
N_{\nu} \subset \overline{\mathcal{M}}_{\chi}(D, 2)^{\bullet} \text { and } \quad W_{\nu} \subset \overline{\mathcal{M}}_{\chi, 1}(D, 2)^{\bullet} .
$$

We denote by $P$ the set of étale double covers of $D$; it is known that $|P|=2^{2 h}$. Thus there are $2^{2 h}$ such components. We let $N_{0}$ be their complement and $W_{0}$ the preimage of $N_{0}$ under $\varphi$ :

$$
N_{0}=\overline{\mathcal{M}}_{\chi}(D, 2)^{\bullet}-\cup_{\nu \in P} N_{\nu}, \quad \text { and } \quad W_{0}=\varphi^{-1}\left(N_{0}\right) .
$$

We let $\pi: \overline{\mathcal{M}}_{\chi, 1}(S, 2)^{\bullet} \rightarrow \overline{\mathcal{M}}_{\chi, 1}(D, 2)^{\bullet}$ be the morphism induced by $p: S \rightarrow D$. We let

$$
M_{a}=\pi^{-1}\left(W_{a}\right), \quad a \in P \cup\{0\} .
$$

Since all $N_{a}, a \in P \cup\{0\}$, are mutually disjoint, the same is true for all $M_{a}$. Thus the localized virtual cycle

$$
\left[\overline{\mathcal{M}}_{\chi, 1}(S, 2)^{\bullet}\right]_{\text {loc }}^{\mathrm{vir}} \in A_{*} \overline{\mathcal{M}}_{\chi, 1}(D, 2)^{\bullet}=\oplus_{a \in P \cup\{0\}} A_{*} W_{a}
$$

decomposes into

$$
\left[\overline{\mathcal{M}}_{\chi, 1}(S, 2)^{\bullet}\right]_{\mathrm{loc}}^{\mathrm{vir}}=\sum_{a \in P \cup\{0\}}\left[M_{a}\right]_{\mathrm{loc}}^{\mathrm{vir}}, \quad\left[M_{a}\right]_{\mathrm{loc}}^{\mathrm{vir}} \in A_{*} W_{a} .
$$

We define the contribution to $\left\langle\tau_{1}(\gamma)\right\rangle_{2[D]}^{S, \bullet}$ from the component $M_{a}$ to be

$$
\left\langle\tau_{1}(\gamma)\right\rangle_{2[D]}^{S, \bullet}\left[M_{a}\right]=\int_{\left[M_{a}\right]_{\mathrm{loc}}^{\mathrm{vir}}} \psi_{1} \mathrm{ev}_{1}^{*}(\gamma) .
$$

(I.e., by replacing $\left[\overline{\mathcal{M}}_{\chi, 1}(S, 2)^{\bullet}\right]_{\text {loc }}^{\mathrm{vir}}$ by $\left[M_{a}\right]_{\text {loc }}^{\mathrm{vir}}$.)

It is easy to see that

$$
\left\langle\tau_{1}(\gamma)\right\rangle_{2[D]}^{S, \bullet}\left[M_{\nu}\right]=(-1)^{h^{0}\left(u^{*} L\right)}\left(-\frac{1}{12}\right), \quad \nu \in P .
$$

Here the sign $(-1)^{h^{0}\left(\nu^{*} L\right)}$ is due to Proposition 3.5 and the factor $-1 / 12$ is from the formula (1.2) for the degree one case. Because exactly $2^{h-1}\left(2^{h}+1\right)$ of the $2^{2 h}$ étale $\nu \in P$ satisfy $h^{0}\left(u^{*} L\right) \equiv h^{0}(L) \bmod 2$, the total contribution

$$
\begin{aligned}
\sum_{\nu \in P}\left\langle\tau_{1}(\gamma)\right\rangle_{2[D]}^{S, \bullet}\left[M_{\nu}\right] & =(-1)^{h^{0}(L)}\left(-\frac{1}{12}\right) \cdot\left(2^{h-1}\left(2^{h}+1\right)-2^{h-1}\left(2^{h}-1\right)\right) \\
& =(-1)^{h^{0}(L)}\left(-\frac{2^{h}}{12}\right) .
\end{aligned}
$$


Therefore to prove (3.5), it suffices to show

Lemma 3.5. The contribution

$$
\left\langle\tau_{1}(\gamma)\right\rangle_{2[D]}^{S, \bullet}\left[M_{0}\right]=(-1)^{h^{0}(L)}\left(\frac{2^{h}}{-3}\right)-(-1)^{h^{0}(L)}\left(-\frac{2^{h}}{12}\right)=(-1)^{h^{0}(L)}\left(-2^{h-2}\right) .
$$

The difficulty in this case is that $\pi_{*} f^{*} L$ is not locally free over $W_{0}$. Were $\pi_{*} f^{*} L$ locally free over $W_{0}$, we could apply Theorem 1.1 to obtain the Lemma. Instead, we will prove this identity by studying its non-locally freeness and comparing it with the twisted GW invariants of $\overline{\mathcal{M}}_{\chi, 1}(D, 2)^{\bullet}$.

Because of the connected component decomposition

$$
\overline{\mathcal{M}}_{\chi, 1}(D, 2)^{\bullet}=\bigsqcup_{a \in P \cup\{0\}} W_{a}
$$

we have

$$
\left[\overline{\mathcal{M}}_{\chi, 1}(D, 2)^{\bullet}\right]^{\mathrm{vir}}=\sum_{a \in P \cup\{0\}}\left[W_{a}\right]^{\mathrm{vir}}
$$

The contributions to the twisted GW invariants from $W_{a}$ are defined with the cycle $\left[\overline{\mathcal{M}}_{\chi, 1}(D, 2)^{\bullet}\right]^{\text {vir }}$ replaced by $\left[W_{a}\right]^{\text {vir }}$.

It is easy to get the contribution to the twisted GW invariant from $W_{0}$. From [3] and [12], it is straightforward to deduce that the degree two GW invariant of $D$ twisted by the top Chern class of $-\pi ! f^{*} L$ is

$$
\left\langle\tau_{1}(\gamma) ; c_{t o p}\left(-\pi_{!} f^{*} L\right)\right\rangle_{2, \chi}^{D, \bullet}=\left(h-\frac{8}{3}\right) 2^{2 h-3}
$$

the contribution to the twisted GW invariant from any of the irreducible components $W_{\nu}$ is $-\frac{1}{12}$. Therefore, the contribution to the twisted GW invariant from $W_{0}$ is

$$
\left\langle\tau_{1}(\gamma) ; c_{t o p}\left(-\pi_{!} f^{*} L\right)\right\rangle_{2, \chi}^{D, \bullet}\left[W_{0}\right]=\left(h-\frac{8}{3}\right) 2^{2 h-3}-2^{2 h}\left(-\frac{1}{12}\right)=(h-2) 2^{2 h-3} .
$$

It remains to determine the difference

$$
\left\langle\tau_{1}(\gamma)\right\rangle_{2[D]}^{S, \bullet}\left[M_{0}\right]-\left\langle\tau_{1}(\gamma) ; c_{t o p}\left(-\pi_{!} f^{*} L\right)\right\rangle_{2, \chi}^{D, \bullet}\left[W_{0}\right]
$$




\section{Explicit virtual CyCle CALCUlation}

After exhausting the general machinery to attack the localized GW invariants, we will turn to an explicit construction to evaluate the term (3.8). We quote the following deformation invariance result.

Proposition $4.1([5])$. The localized $G W$ invariants of the spin surface $S \rightarrow D$ are deformation invariant under the deformation of spin pair $(D, L)$ (i.e. such that $\left.L^{\otimes 2} \cong K_{D}\right)$.

This is proved in [5], and will appear in the proof of the degeneration formula of GW invariants for spin surfaces [7].

Because of this invariance, we can replace the spin pair $(L, D)$ by another pair that lies in the same connected component of the space of all spin pairs. As is well known, two spin pairs $(L, D)$ and $\left(L^{\prime}, D^{\prime}\right)$ lie in the same connected component if the parity of $L$, which is $h^{0}(L) \bmod 2$, equals the parity of $L^{\prime}$. Applying the invariance result, we can assume without loss of generality that $D$ is a hyperelliptic curve.

We now set up the stage for our calculation. Our first step is to identify the obstruction sheaf of $M_{0}$. By definition, the obstruction sheaf $\mathcal{O} b_{M_{0}}$ is the cokernel of the homomorphism

$$
\mathcal{E} x t_{\tilde{\pi}}^{1}\left(\Omega_{\tilde{\mathcal{C}}}(\tilde{s}), \mathcal{O}_{\tilde{\mathcal{C}}}\right) \longrightarrow R^{1} \tilde{\pi}_{*} \tilde{f}^{*} T_{S}
$$

Here $(\tilde{\pi}, \tilde{f}, \tilde{s}, \tilde{\mathcal{C}})$ and $(\pi, f, s, \mathcal{C})$ are the universal families of $M_{0} \subset \overline{\mathcal{M}}_{\chi, 1}(S, 2)^{\bullet}$ and $W_{0} \subset \overline{\mathcal{M}}_{\chi, 1}(D, 2)^{\bullet}$, respectively. Here $\tilde{s}$ and $s$ are the sections of the marked points, viewed as divisors in $\tilde{\mathcal{C}}$ and $\mathcal{C}$ respectively.

Composing (4.1) with the tautological $R^{1} \tilde{\pi}_{*} \tilde{f}^{*} T_{S} \rightarrow R^{1} \tilde{\pi}_{*} \tilde{f}^{*} p^{*} T_{D}$, we obtain

$$
\mathcal{E} x t_{\tilde{\pi}}^{1}\left(\Omega_{\tilde{\mathcal{C}}}(\tilde{s}), \mathcal{O}_{\tilde{\mathcal{C}}}\right) \longrightarrow R^{1} \tilde{\pi}_{*} \tilde{f}^{*} p^{*} T_{D}
$$

We claim that it is surjective. Using $\tilde{\mathcal{C}}=\mathcal{C} \times{ }_{W_{0}} M_{0},(4.2)$ is the pullback to $M_{0}$ of the similarly defined $\mathcal{E} x t_{\pi}^{1}\left(\Omega_{\mathcal{C}}(s), \mathcal{O}_{\mathcal{C}}\right) \rightarrow R^{1} \pi_{*} f^{*} T_{D}$, which is surjective since $(f, \pi): \mathcal{C} \rightarrow D \times W_{0}$ is finite and surjective. Let $\mathcal{K}$ be the kernel of (4.2) which is locally free on $W_{0}$.

Lemma 4.2. The sheaf $R^{1} \tilde{\pi}_{*} \tilde{f}^{*} T_{S}$ fits into the short exact sequence

$$
0 \longrightarrow R^{1} \tilde{\pi}_{*} \tilde{f}^{*} p^{*} L \longrightarrow R^{1} \tilde{\pi}_{*} \tilde{f}^{*} T_{S} \longrightarrow R^{1} \tilde{\pi}_{*} \tilde{f}^{*} p^{*} T_{D} \longrightarrow 0
$$


the composite $\mathcal{K} \rightarrow \mathcal{E} x t_{\tilde{\pi}}^{1}\left(\Omega_{\tilde{\mathcal{C}}}(\tilde{s}), \mathcal{O}_{\tilde{\mathcal{C}}}\right) \rightarrow R^{1} \tilde{\pi}_{*} \tilde{f}^{*} T_{S}$ lifts to a unique

$$
\mathcal{K} \longrightarrow R^{1} \tilde{\pi}_{*} \tilde{f}^{*} p^{*} L
$$

its cokernel is the obstruction sheaf $\mathcal{O} b_{M_{0}}$.

Proof. Using the exact sequence $0 \rightarrow p^{*} L \rightarrow T_{S} \rightarrow p^{*} T_{D} \rightarrow 0$, we obtain the long exact sequence

$$
\longrightarrow \tilde{\pi}_{*} \tilde{f}^{*} T_{S} \stackrel{a_{0}}{\longrightarrow} \tilde{\pi}_{*} \tilde{f}^{*} p^{*} T_{D} \stackrel{a_{1}}{\longrightarrow} R^{1} \tilde{\pi}_{*} \tilde{f}^{*} p^{*} L \longrightarrow R^{1} \tilde{\pi}_{*} \tilde{f}^{*} T_{S} \longrightarrow .
$$

Suppose $a_{1}$ (in the sequence) is trivial, then $\mathcal{K} \rightarrow R^{1} \tilde{\pi}_{*} \tilde{f}^{*} T_{S}$ lifts to $\mathcal{K} \rightarrow$ $R^{1} \tilde{\pi}_{*} \tilde{f}^{*} p^{*} L$; since (4.2) is surjective, $\mathcal{O} b_{M_{0}}$ is the cokernel of (4.3).

We now prove $a_{1}=0$. In case $h=g(D) \geq 2$, we have $\tilde{\pi}_{*} \tilde{f}^{*} T_{D}=0$, thus $a_{1}=0$. In case $h=1$, then $T_{D}=\mathcal{O}_{D}$ and $\tilde{\pi}_{*} \tilde{f}^{*} T_{D}=\mathcal{O}_{M_{0}}$ is generated by a one-parameter family of automorphisms $\eta_{t}: D \rightarrow D, \eta_{0}=$ id. Since $L^{\otimes 2} \cong \mathcal{O}_{D}$, $\left(\eta_{t}^{*} L\right)^{\otimes 2} \cong \mathcal{O}_{D}$. Thus $\eta_{t}^{*} L \cong L$, and hence $\eta_{t}$ lift to $\tilde{\eta}_{t}: S \rightarrow S$, which generates a section in $\tilde{\pi}_{*} \tilde{f}^{*} T_{S}$ that surjects onto $\tilde{\pi}_{*} \tilde{f}^{*} p^{*} T_{D}$. This shows that $a_{0}$ is surjective, hence $a_{1}=0$. The case $h=0$ is similar.

By the theory of (localized) virtual cycles, to get (localized) GW invariants, we need to identify the intrinsic virtual normal cone

$$
\mathbf{C}_{M_{0}} \subset h^{1} / h^{0}\left(R \tilde{\pi}_{*} \tilde{f}^{*} T_{S}\right)=h^{1} / h^{0}\left(R \tilde{\pi}_{*} \tilde{f}^{*} p^{*} L\right)
$$

(Here the identity follows from the proof of Lemma 4.2.) Over the locus $U \subset W_{0}$ where $R^{1} \pi_{*} f^{*} L$ is locally free, the moduli stack $M_{0} \times_{W_{0}} U$ is smooth, and then $\mathbf{C}_{M_{0}} \times_{W_{0}} U$ is the zero section of $h^{1} / h^{0}\left(R \tilde{\pi}_{*} \tilde{f}^{*} p^{*} L\right) \times_{W_{0}} U$. This shows that the zero section is part of $\mathbf{C}_{M_{0}}$, and of multiplicity one.

The remainder components of $\mathbf{C}_{M_{0}}$ support over the complement of $M_{0} \times{ }_{W_{0}} U$. These components can be analyzed by knowing the stack structure of $M_{0}$; this will be the main technical part of this section.

Finally, we need to intersect $\mathbf{C}_{M_{0}}$ by applying the localized Gysin map. Applying the localized Gysin map to the zero section part of $\mathbf{C}_{M_{0}}$ amounts to determining the degree of the sheaf $R^{1} \pi_{*} f^{*} L$ on $W_{0}$, which is carried out by the twisted GW invariant of $D$ (see the argument before (4.22)). Applying the localized Gysin map to other components of $\mathbf{C}_{M_{0}}$ is carried out by direct calculation; this is possible since the locus where $R^{1} \pi_{*} f^{*} L$ fails to be locally free is finite. 
This calculation shows that the "top dimensional contribution" to the localized GW invariant comes from the twisted GW invariant. To get the full invariant, one approach is to pinpoint the "lower dimensional" contributions. The method presented in this paper is our answer to this question. It is limited; nevertheless we hope that it will shed lights on finding a more powerful machinery.

We now carry out the details. As mentioned, we assume $D$ is hyperelliptic, we let $\delta: D \rightarrow \mathbb{P}^{1}$ be its associated double cover; we will cut down the space $W_{0}$ by the insertion $\tau_{1}(\gamma)=\psi_{1} e v_{1}^{*}(\gamma)$.

We first describe the space $N_{0}$. An easy argument shows that closed points of $N_{0}$ are double covers $u: C \rightarrow D$ branched at two points $q_{1}, q_{2} \in D$. It is known that such map is characterized by a line bundle $\xi$ on $D$ satisfying

$$
\xi^{\otimes 2} \cong \mathcal{O}_{D}\left(q_{1}+q_{2}\right) ;
$$

the curve $C$ is a subscheme of the total space of $\xi$ defined by $\left\{t \in \xi \mid t^{2}=v\right\}$ for $v$ a non-trivial section in $H^{0}\left(\mathcal{O}_{D}\left(q_{1}+q_{2}\right)\right)$ vanishing at $q_{1}+q_{2}$; the map $u$ is the one induced by the projection $\xi \rightarrow D$. Further, a direct obstruction analysis shows that $\overline{\mathcal{M}}_{\chi}(D, 2)^{\bullet}$ is smooth near such double covers $[u]$. Thus this description gives the scheme structure of $N_{0}: N_{0}$ is a smooth two dimensional DM stack, with $\mathbb{Z}_{2}$ automorphism group at every point. Consequently, $W_{0}$ is the quotient by $\mathbb{Z}_{2}$ of the total space of the universal curve of $\overline{\mathcal{M}}_{\chi}(D, 2)^{\bullet}$ over $N_{0}$; it is a smooth three dimensional DM stack, and the only points with $\mathbb{Z}_{2}$ automorphism groups are those $[u]$ whose two branched points and the marked point coincide.

We now let

$$
f: \mathcal{C} \rightarrow D, \quad \pi: \mathcal{C} \rightarrow W_{0}, \quad s: W_{0} \rightarrow \mathcal{C} \text { the section of marked points }
$$

be the universal family of $W_{0}$; we let $\mathrm{ev}_{1}: W_{0} \rightarrow D$ as before be the evaluation morphism. We can cut down $W_{0}$ by $\psi_{1} e v_{1}^{*}(\gamma)$ as follows: we pick a general point $q \in D$ and form the subscheme $e v_{1}^{-1}(q)$; it is a Cartier divisor of $W_{0}$ and it represents the class $e v_{1}^{*}(\gamma)$.

For $\psi_{1}$, the natural homomorphism $s^{*} f^{*} \Omega_{D} \rightarrow s^{*} \omega_{\mathcal{C} / W_{0}}$ induces a section

$$
\phi \in \Gamma\left(W_{0}, s^{*}\left(\omega_{\mathcal{C} / W_{0}} \otimes f^{*} \Omega_{D}^{\vee}\right)\right) .
$$

This section vanishes at $[u]=\left(\xi, q_{1}+q_{2}\right) \in W_{0} \cap e v_{1}^{-1}(q)$ if and only if the marked point $s_{u}$ of $[u]$ is one of the two branch points $q_{1}$ and $q_{2}$. 
We introduce

$$
Z=\{\phi=0\}, \quad Z_{q}=Z \cap e v_{1}^{-1}(q), \quad M_{0, q}=M_{0} \times_{W_{0}} Z_{q} .
$$

Then the cycle contributing to $\left\langle\tau_{1}(\gamma)\right\rangle_{2[D]}^{S, \bullet}\left[M_{0}\right]$ lies inside $M_{0, q}$.

Our next step is to investigate the stack structure of $M_{0}$ near $M_{0, q}$. According to Lemma 2.1, it suffices to investigate the locus $\Lambda \subset W_{0}$ over which the sheaf $R^{1} \pi_{*} f^{*} L$ is non-locally free. For a double cover $u: C \rightarrow D$ given by $\left(\xi, q_{1}+q_{2}\right)$, since

$$
H^{0}\left(C, u^{*} L\right)=H^{0}(D, L) \oplus H^{0}\left(D, L \otimes \xi^{-1}\right),
$$

it is easy to see that $R^{1} \pi_{*} f^{*} L$ is non-locally free exactly along the locus

$$
\Lambda=\left\{u=\left(\xi, q_{1}+q_{2}\right) \in W_{0} \mid H^{0}\left(L \otimes \xi^{-1}\right) \neq 0\right\} .
$$

Here the marked point $s_{u} \in C$ of $u$ is implicitly understood.

Let $u=\left(\xi, q_{1}+q_{2}\right) \in \Lambda$, and let

$$
\eta \neq 0 \in H^{0}\left(D, L \otimes \xi^{-1}\right) .
$$

We denote $H=\delta^{*} \mathcal{O}_{\mathbb{P}^{1}}(1)$. As $L^{2} \cong K_{D}=(h-1) H$ and $\xi^{\otimes 2}=\mathcal{O}\left(q_{1}+q_{2}\right)$, we have

$$
(h-1) H=q_{1}+q_{2}+2 \eta^{-1}(0),
$$

which, because $D$ is hyperelliptic, is possible only if

$$
\text { either } q_{1}=q_{2}, \quad \text { or } q_{1}=\bar{q}_{2},
$$

Here we use $\bar{q}_{2}$ to denote the point conjugate to $q_{2}$ with respect to $\delta: D \rightarrow \mathbb{P}^{1}$. In particular, when $u \in \Lambda \cap Z_{q}$, one of $q_{i}$ must be $q$; thus $\Lambda \cap Z_{q}$ is finite.

Let

$$
R=\left\{u=\left(\xi, q_{1}+q_{2}\right) \in W_{0} \mid q_{1}=\bar{q}_{1} \text { or } q_{2}=\bar{q}_{2}\right\} .
$$

Since $q \in D$ is general, $\Lambda \cap Z_{q} \cap R=\emptyset$. As the desired contribution lies in $M_{0, q}$, we only need to study the set

$$
\Lambda^{\prime}=\left\{u=\left(\xi, q_{1}+q_{2}\right) \in \Lambda-R \mid q_{1}=\bar{q}_{2}\right\}, \quad \Lambda^{\prime \prime}=\left\{u \in \Lambda-R \mid q_{1}=q_{2}\right\}
$$

Note that $\Lambda^{\prime}$ and $\Lambda^{\prime \prime}$ is a partition of $\Lambda-R$. 
It is easy to identify the set $\Lambda^{\prime}$. Let $u=(\xi, q+\bar{q})$ be any point in this set. Since $q+\bar{q}=H,(4.8)$ reduces to $2 \eta^{-1}(0)=(h-2) H$. Let

$$
r=h^{0}\left(L \otimes \xi^{-1}\right)-1=h^{1}\left(L \otimes \xi^{-1}\right)-2,
$$

which ranges between 0 and $(h-2) / 2$. Since $D$ is hyperelliptic, we can write the effective divisor

$$
\eta^{-1}(0)=p_{1}+\cdots+p_{h-2}, p_{i} \in C
$$

so that

(a). $p_{i+r}=\bar{p}_{i}$ for $i \leq r$;

(b). all $p_{j}$ for $j \geq 2 r+1$ are distinct ramification points of $\delta: D \rightarrow \mathbb{P}^{1}$.

The $r$ in (4.10) decomposes $\Lambda^{\prime}$ into disjoint union of $\Lambda_{r}^{\prime}$ for $0 \leq r \leq(h-2) / 2$. Since

$$
\xi=L\left(-p_{1}-\cdots-p_{h-2}\right)=L-r H-p_{2 r+1}-\cdots-p_{h-2},
$$

elements in $\Lambda_{r}^{\prime}$ are uniquely determined by the distinct ramified points $p_{j>2 r}$ of $\delta: D \rightarrow \mathbb{P}^{1}$.

Looking at the intersection $\Lambda^{\prime} \cap Z_{q}$, since the branch points of $u \in \Lambda^{\prime} \cap Z_{q}$ must be $q_{1}+\bar{q}_{1}=q+\bar{q}$, and the marked point of $u$ must be $q$, (noting $q \neq \bar{q}$,) we conclude that $\Lambda_{r}^{\prime} \cap Z_{q}$ consists of $\left(\begin{array}{c}2 h+2 \\ h-2-2 r\end{array}\right)$ elements, where $2 h+2$ is the number of ramification points of $\delta$.

Claim 4.3. The scheme structure of $M_{0, q}$ near the fiber over $u=(\xi, q+\bar{q}) \in \Lambda_{r}^{\prime}$ is (analytically) isomorphic to

$$
\mathbf{A}^{l} \times\left\{z w_{1}=z^{3} w_{2}=\cdots=z^{2 r+1} w_{r+1}=0\right\} \subset \mathbf{A}^{l+r+2}, \quad l=h^{0}(L)
$$

near $\mathbf{A}^{l} \times\{0\} \subset \mathbf{A}^{l+r+2}$, with automorphisms $\mathbb{Z}_{2}$ acting trivially at every point.

Proof. Since $Z_{q}$ is smooth near $u$, the local defining equation of $M_{0, q}$ is determined by a locally free resolution of $\bar{\pi}_{!} \bar{f}^{*} L$ for $\bar{f}: \overline{\mathcal{C}} \rightarrow D$ and $\bar{\pi}: \overline{\mathcal{C}} \rightarrow Z_{q}$ the restrictions of $f$ and $\pi$ to $Z_{q}$. On the other hand, from (4.7), we see immediately that $\bar{\pi}_{*} \bar{f}^{*} L=H^{0}(L) \otimes \mathcal{O}_{Z_{q}}$. By Riemann-Roch, away from $M_{0, q} \times_{W_{0}} \Lambda, R^{1} \bar{\pi}_{*} \bar{f}^{*} L$ is locally free and has rank $l+1$. Therefore, $R^{1} \bar{\pi}_{*} \bar{f}^{*} L$ is a direct sum of its torsion free part $\mathcal{R}$ and its torsion part.

Since $h^{1}\left(L \otimes \xi^{-1}\right)=r+2$ by Riemann-Roch [1], in a formal neighborhood of $u \in Z_{q}$ with $z$ the local coordinate of $Z_{q}$ at $u$, we can find positive integers 
$\alpha_{1} \leq \alpha_{2} \leq \cdots \leq \alpha_{r+1}$ and express the torsion part of $R^{1} \bar{\pi}_{*} \bar{f}^{*} L$ as

$$
\mathbb{C}[[z]] /\left(z^{\alpha_{1}}\right) \oplus \cdots \oplus \mathbb{C}[[z]] /\left(z^{\alpha_{r+1}}\right) .
$$

Thus a (locally free) resolution of $\bar{\pi}_{!} \bar{f}^{*} L$ can be chosen as

$$
\operatorname{diag}\left(z^{\alpha_{1}}, \cdots, z^{\alpha_{r+1}}\right): \bigoplus_{i=1}^{r+1} \mathcal{O} \longrightarrow\left(\bigoplus_{i=1}^{r+1} \mathcal{O}\right) \oplus \mathcal{R}
$$

By Lemma 2.1, if we let $\left(z_{1}, \cdots, z_{l}, w_{1}, \cdots, w_{r+1}\right)$ be the coordinates for the vector space $H^{0}\left(C, f^{*} L\right)=H^{0}(L) \oplus H^{0}\left(L \otimes \xi^{-1}\right)$, the local defining equation of $M_{0, q}$ near fibers over $u$ can be chosen as

$$
z^{\alpha_{1}} w_{1}=z^{\alpha_{2}} w_{2}=\cdots=z^{\alpha_{r+1}} w_{r+1}=0 .
$$

It remains to determine the integers $\alpha_{i}$. For this we need to investigate whether a line $\mathbb{C} \eta \subset H^{0}\left(L \otimes \xi^{-1}\right)$ can be extended to a submodule

$$
\mathbb{C}[z] /\left(z^{n}\right) \subset \pi_{*} f^{*} L / z^{n} \pi_{*} f^{*} L .
$$

Let

$$
H^{0}\left(L \otimes \xi^{-1}\right)=F_{r+1} \supseteq F_{r} \supseteq \cdots \supseteq F_{1} \supseteq F_{0}=\{0\}
$$

be a flag with $F_{r+1-k}=H^{0}\left(L \otimes \xi^{-1}(-k q)\right)$. It is a complete flag since $h^{0}(L \otimes$ $\left.\xi^{-1}(-k q)\right)=\max (r+1-k, 0)$, using (4.11). For $0 \leq k \leq r$, let $\eta \in F_{r+1-k}-F_{r-k}$ be a general element and $\left(p_{i}\right)$ be the zeros of $\eta$. Then after reshuffling if necessary, we have

$p_{1}=\cdots=p_{k}=q, p_{r+1}=\cdots=p_{r+k}=\bar{q}, p_{k+1}, \cdots, p_{r}, p_{r+k+1}, \cdots, p_{2 r} \notin\{q, \bar{q}\}$.

Suppose $\eta$ fits into a $\mathbb{C}[z] /\left(z^{n}\right)$-modules as in (4.14), then there are morphisms

$$
\mathbf{q}, \mathbf{q}^{\prime} \text { and } \mathbf{p}_{i}: \operatorname{Spec} \mathbb{C}[z] /\left(z^{n}\right) \longrightarrow D,
$$

which extend the points $q, \bar{q}$ and $p_{i}$ respectively, such that $\mathbf{q}$ is constant, $\mathbf{q}^{\prime}$ has non-vanishing first order variation, and viewing $\mathbf{q}, \mathbf{q}^{\prime}, \mathbf{p}_{i}$ as families of divisors on $D$ parameterized by $\operatorname{Spec} \mathbb{C}[z] /\left(z^{n}\right)$, the relation (4.8) holds as $\operatorname{Spec} \mathbb{C}[z] /\left(z^{n}\right)$ families of Cartier divisors:

$$
(h-1) H=\mathbf{q}+\mathbf{q}^{\prime}+2 \mathbf{p}_{1}+\cdots+2 \mathbf{p}_{h-2} .
$$

Because $\bar{q}$ is not a branch point of $\delta: D \rightarrow \mathbb{P}^{1}$, our local coordinate $z$ for $Z_{q}$ at $u$ can be thought of as a local coordinate for $D$ near $\bar{q}$ and also a local coordinate for $\mathbb{P}^{1}$ via $\delta$. Without loss of generality, we can choose $\mathbf{q}^{\prime}$ so that $\delta \circ \mathbf{q}^{\prime}(z)=z$. 
Suppose $\delta \circ \mathbf{p}_{i}=p_{i}(z)$. Then the above identity on divisors over $\operatorname{Spec} \mathbb{C}[z] /\left(z^{n}\right)$ is equivalent to

$$
w \prod_{i=1}^{k}\left(w-p_{i}(z)\right)^{2} \equiv(w-z) \prod_{i=1}^{k}\left(w-p_{r+i}(z)\right)^{2} \bmod z^{n},
$$

for a formal variable $w$.

Shortly, we shall show that (4.15) is solvable if and only if $n \leq 2 k+1$. Once this is done, we see that because $\operatorname{dim} F_{k}=k$, we have $\alpha_{1}=1, \alpha_{2}=3, \ldots, \alpha_{r}=2 r+1$. This will provide us the structure result of $M_{0, q}$ over an element in $\Lambda_{r}^{\prime}$.

We now prove that (4.15) is solvable if and only if $n \leq 2 k+1$. We first compare the constant coefficients in $w$ of (4.15) to obtain

$$
z \prod_{i=1}^{k} p_{r+i}^{2}(z) \equiv 0 \quad \bmod z^{n}
$$

To proceed, we shall show that for $n=2 k+1$, (4.15) holds if and only if $p_{r+i}(z) \equiv_{\left(z^{2}\right)} c_{i} z$ for uniquely determined complex numbers $c_{i} \neq 0,1 \leq i \leq k$. (Here we use $\equiv_{\left(z^{n}\right)}$ to mean equivalence modulo $z^{n}$.) Together with (4.16), this immediately implies the claim.

Let $n=2 k+1$; let $f, g$ and $h$ be defined by

$$
f=\prod_{i=1}^{k}\left(1-p_{i}(z) / w\right)=\sum_{j=0}^{k} A_{j} w^{-j}, \quad g=\prod_{i=1}^{k}\left(1-p_{r+i}(z) / w\right)=\sum_{j=0}^{k} B_{j} w^{-j}
$$

and $h=\sqrt{1-z / w} \cdot g=\sum_{j=0}^{\infty} C_{j} w^{-j}$, with $A_{i}, B_{i}$ and $C_{i}$ analytic functions of $z$. Dividing (4.15) by $w^{2 k+1}$, we see immediately that that $C_{j} \equiv_{\left(z^{n}\right)} A_{j}$ for $0 \leq j \leq k$ and that $h^{2}-f^{2}=2 f(h-f)+(h-f)^{2}$ has no terms $w^{-j}$ with $j \leq 2 k$, modulo $z^{n}$ as usual. Since $f$ is monic and $h-f$ has no terms $w^{-j}$ with $j \leq k, h-f$ has no terms $w^{-j}$ with $j \leq 2 k$. This implies that $C_{j} \equiv_{\left(z^{n}\right)} 0$ for $k<j \leq 2 k$. If we let $\sqrt{1-z / w}=\sum D_{j} w^{-j}$, we obtain

$$
0 \equiv_{\left(z^{n}\right)} C_{j}=\sum_{i=0}^{k} D_{j-i} B_{i}=D_{j}+\sum_{j=1}^{k} D_{j-i} B_{i}
$$

for $k<j \leq 2 k$, and thus we have a matrix equation

$$
\left(G_{1} G_{2} \cdots G_{k}\right) B \equiv_{\left(z^{n}\right)}-G_{k+1},
$$


where $G_{j}$ is the column vector $\left(D_{j}, D_{j+1}, \cdots, D_{j+k-1}\right)^{t}$ and $B$ is the column vector $\left(B_{k}, B_{k-1}, \cdots, B_{1}\right)^{t}$. Since $D_{j}=-2^{1-2 j} \frac{1}{j}\left(\begin{array}{c}2 j-2 \\ j-1\end{array}\right) z^{j}$, the determinant of the Hankel matrix $\left(G_{1} G_{2} \cdots G_{k}\right)$ is $\left[(-1)^{k} / 2^{2 k^{2}-k}\right] z^{k^{2}}$ and the determinant of $\left(G_{2} G_{3} \cdots G_{k+1}\right)$ is $\left[(-1)^{k} / 2^{2 k^{2}+k}\right] z^{k^{2}+k}$. (See [13] for the computation of these Hankel determinants.) To prove the solvability of (4.15) for $n=2 k+1$, we can replace $\equiv_{\left(z^{n}\right)}$ by the honest equality. By Cramer's rule, the matrix equation $\left(G_{1} G_{2} \cdots G_{k}\right) B=-G_{k+1}$ has a unique solution $B_{k}=(-4)^{-k} z^{k}$ and $B_{j}=\beta_{j} z^{j}$ for some $\beta_{j} \in \mathbb{C}, j \leq k$. The equation $A_{j}=C_{j}$ for $j \leq k$ implies $A_{j}=\gamma_{j} z^{j}$ for some $\gamma_{j} \in \mathbb{C}$. Therefore $p_{r+i}(z)=c_{i} z$ for some $c_{i} \neq 0$ solves (4.15) and (4.16).

To see the insolvability of (4.15) for $n>2 k+1$, we observe from (4.17) that the least order terms of $B_{j}$ are uniquely determined as above and thus $p_{r+i}(z) \equiv_{\left(z^{2}\right)}$ $c_{i} z$ for $c_{i} \neq 0$. Then (4.16) cannot be satisfied. This completes our description of the formal neighborhoods of exceptional points in $\Lambda_{r}^{\prime}$.

The structure of $\Lambda^{\prime \prime}$ is similar.

Claim 4.4. The intersection $\Lambda^{\prime \prime} \cap Z_{q}$ is the disjoint union of $\Lambda_{r}^{\prime \prime}$ for $0 \leq r \leq \frac{h-3}{2}$; each $\Lambda_{r}^{\prime \prime}$ has $\left(\begin{array}{c}2 h+2 \\ h-3-2 r\end{array}\right)$ elements; the local defining equation for the stack $M_{0, q}$ near a fiber over a point in $\Lambda_{r}^{\prime \prime}$ is (analytically) isomorphic to

$$
\mathbf{A}^{l} \times\left\{z^{2} w_{1}=z^{4} w_{2}=\cdots=z^{2(r+1)} w_{r+1}=0\right\} \subset \mathbf{A}^{l+r+2}
$$

near $\mathbf{A}^{l} \times\{0\} \subset \mathbf{A}^{l+r+2}$, with authmorphisms $\mathbb{Z}_{2}$ acting trivially at every point.

Proof. The proof is parallel, and will be omitted.

These two claims immediately give us

Corollary 4.5. The stack $M_{0, q}$ is the union of closed substacks $V_{0}$ and $V_{u}$, where $u$ are indexed by $u \in \Lambda \cap Z_{q}$ as follows:

(a) $V_{0}$ is the vector bundle $H^{0}(L) \times Z_{q}$ over $Z_{q}$;

(b) for $u \in \Lambda^{\prime} \cap Z_{q}$, following the notation of Claim 4.3, $V_{u}$ is

$$
\mathbf{A}^{l} \times\left\{z w_{1}=z^{3} w_{2}=\cdots=z^{2 r+1} w_{r+1}=z^{2 r+1}=0\right\} \subset \mathbf{A}^{l+r+2} ;
$$

(c) for $u \in \Lambda^{\prime \prime} \cap Z_{q}$, following the notation of Claim 4.4, $V_{u}$ is

$$
\mathbf{A}^{l} \times\left\{z^{2} w_{1}=z^{4} w_{2}=\cdots=z^{2(r+1)} w_{r+1}=z^{2(r+1)}=0\right\} \subset \mathbf{A}^{l+r+2} .
$$

The stack $M_{0, q}$ has automorphism group $\mathbb{Z}_{2}$ acting trivially at every point. 
We let $B \subset \mathcal{C}$ be the branch locus of the morphism $f: \mathcal{C} \rightarrow W_{0} \times D$; let $R=f(B) \subset W_{0} \times D$ be its image, and let $\Xi$ be the line bundle on $W_{0} \times D$ so that $\Xi^{\otimes 2} \cong \mathcal{O}_{W_{0} \times D}(R)$. Then

$$
R^{1} \tilde{\pi}_{*} \tilde{f}^{*} p^{*} L \cong \phi^{*} R^{1} \pi_{W_{0} *} \pi_{D}^{*} L \oplus \phi^{*} R^{1} \pi_{W_{0} *}\left(\pi_{D}^{*} L \otimes \Xi^{-1}\right) .
$$

Here $\pi_{W_{0}}$ and $\pi_{D}$ are the projections of $W_{0} \times D$, and $\phi: M_{0} \rightarrow W_{0}$ is the projection. Of the two, $R^{1} \pi_{W_{0} *} \pi_{D}^{*} L=H^{1}(L) \otimes \mathcal{O}_{W_{0}}$ is locally free of rank $l=h^{0}(L)$.

We next pick a vector bundle $F$ on $W_{0}$ together with a surjective homomophism $\mathcal{O}_{W_{0}}(F) \rightarrow R^{1} \pi_{W_{0} *}\left(\pi_{D}^{*} L \otimes \Xi^{-1}\right)$. We let

$$
\mathcal{O}_{W_{0}}(E)=H^{1}(L) \otimes \mathcal{O}_{W_{0}} \oplus \mathcal{O}_{W_{0}}(F) .
$$

By Lemma 4.2, $\mathcal{O} b_{M_{0}}$ is a quotient sheaf of $\mathcal{O}_{W_{0}}(E)$. We let $\tilde{F}=\phi^{*} F$ and $\tilde{E}=\phi^{*} E$. We let $Q \subset Z_{*} \tilde{E}$ be the virtual normal cone cycle that is the pullback of the intrinsic normal come of $M_{0}$; we let $\bar{\sigma}: \tilde{E} \rightarrow \mathcal{O}_{M_{0}}$ be the homomorphism induced by the holomorphic two-form $\theta$ on $S$. Then we know that $Q$ lies in the union of $\left.\tilde{E}\right|_{W_{0}}$ and $\operatorname{ker}\left\{\left.\bar{\sigma}\right|_{M_{0}-W_{0}}\right\}$. Applying the localized Gysin map [6], $s_{\tilde{E}, \bar{\sigma}}(Q) \in A_{*} W_{0}$ and

$$
\left\langle\tau_{1}(\gamma)\right\rangle_{2[D]}^{S, \bullet}\left[M_{0}\right]=\int_{S_{\tilde{E}, \bar{\sigma}}^{!}(Q)} \psi \cdot \mathrm{ev}^{-1}(q), \quad q \in D .
$$

Recall that $Z_{q} \subset W_{0}$ is defined in (4.6). We call $q \in D$ general if no irreducible component of $Q$ lies entirely over $M_{0, q}$. Since the obstruction to deforming pointed stable maps does not depend on the location of the marked points, the cycle $Q$ is the pullback of a cycle on $\overline{\mathcal{M}}_{\chi}(S, 2)^{\bullet}$. Thus, for general $q \in D$,

$$
Q_{q}=Q \times_{M_{0}} M_{0, q} \in Z_{*} M_{0, q}
$$

is well-defined and for $\tilde{E}_{q}=\left.\tilde{E}\right|_{M_{0, q}}$ and $\bar{\sigma}_{q}=\left.\bar{\sigma}\right|_{M_{0, q}}$,

$$
\left\langle\tau_{1}(\gamma)\right\rangle_{2[D]}^{S, \bullet}\left[M_{0}\right]=\int_{S_{\tilde{E}_{q}, \bar{\sigma} q}^{!}\left(Q_{q}\right)} 1 .
$$

The description of $Q_{q}$ is given by the following claim. Let $R_{q}$ be the torsion free part of $R^{1} \pi_{*} f^{*} L \otimes_{\mathcal{O}_{W_{0}}} \mathcal{O}_{Z_{q}}$. Since $Z_{q}$ is smooth of dimension one, it is locally free. Let $\phi_{q}: M_{0, q} \rightarrow Z_{q} \subset W_{0}$ be the projection. The quotient $\left.R^{1} \pi_{*} f^{*} L\right|_{Z_{q}} \rightarrow R_{q}$ pullbacks to

$$
\left.\tilde{E}_{q} \longrightarrow R^{1} \tilde{\pi}_{*} \tilde{f}^{*} p^{*} L\right|_{M_{0, q}} \longrightarrow \phi_{q}^{*} R_{q}
$$


Following the notation of Corollary 4.5, for $u \in \Lambda \cap Z_{q}$ we let

$$
V_{u, i}=\mathbf{A}^{l} \times\left\{w_{1}=\cdots=w_{i+1}=0\right\} \subset V_{u} .
$$

Let $m=\operatorname{rank} E$. We have

Claim 4.6. The cycle $Q_{q}$ has a decomposition $Q_{q}=T_{0}+\sum_{u \in \Lambda \cap Z_{q}} m_{u, i} T_{u, i}$ :

(1) $T_{0}$ is the total space of the bundle $\operatorname{ker}\left\{\tilde{E}_{q} \rightarrow \phi_{q}^{*} R_{q}\right\} \times{ }_{Z_{q}} V_{0}$ over $V_{0}$;

(2) for $u \in \Lambda^{\prime} \cap Z_{q}, Q_{q}$ has irreducible components $T_{u, i}$ lying over $V_{u}, 0 \leq$ $i \leq r ; T_{u, i}$ is a rank $m+i-(l+r+1)$ subbundle of $\left.\tilde{E}\right|_{V_{u, i}}, m_{u, i}=2 i+1$;

(3) for $u \in \Lambda^{\prime \prime} \cap Z_{q}$, $Q_{q}$ has irreducible components $T_{u, i}$ lying over $V_{u}, 0 \leq$ $i \leq r ; T_{u, i}$ is a rank $m+i-(l+r+1)$ subbundle of $\left.\tilde{E}\right|_{V_{u, i}}, m_{u, i}=2 i+2$.

Proof. The key is that $M_{0, q}$ near $M_{0, q} \times_{Z_{q}} u$ for $u \in \Lambda^{\prime} \cap Z_{q}$ has local defining equation given by Claim 4.3; the normal bundle to the subscheme in (4.12) is the union of $\mathbf{A}^{l+1}$ (= $\mathbf{A}^{l} \times \mathbf{A}^{1}$, where $\mathbf{A}^{1}$ is the $z$-variable part, ) with $r+1$ additional irreducible components over $V_{u}$ (i.e. lies over $z=0$ ): the $i$-th component is a subbundle of $\mathbf{A}^{r+1} \times V_{u, i} \rightarrow V_{u, i}$, of fiber rank $i+1$, and of multiplicity $2 i+1$.

Let $m=\operatorname{rank} E$. Since $Q_{q}$ has pure dimension $m$, and since $\operatorname{dim} V_{u, i}=l+r+$ $1-i, Q_{q}$ has an irreducible component, that is a rank $m+i-r-l-1$ vector bundle over $V_{u, i}$. It has multiplicity $m_{u, i}=2 i+1$.

The same argument applied to $u \in \Lambda^{\prime \prime} \cap L_{q}$ using the explicit defining equation in Claim 4.4 proves item (3) in the statement of the claim.

It remains to show that the remaining part of $Q_{q}$ is (1). Indeed, since $M_{0, q}-$ $M_{0, q} \times Z_{q} \Lambda$ is smooth, the cone cycle $Q_{q}$ over $M_{0, q}-M_{0, q} \times Z_{q} \Lambda$ is the kernel bundle $\tilde{E}_{q} \rightarrow \phi_{q}^{*} R_{q}$. Using the description of the normal cone to (4.12) and (4.18), we know that near fibers over $u \in \Lambda \cap Z_{q}$, the cycle $Q_{q}$ consists of parts listed in (2) and (3) plus a vector bundle over $V_{0}$. Since this bundle is the kernel bundle of $\tilde{E}_{q} \rightarrow \phi_{q}^{*} R_{q}$ at general points of $V_{0}$, and since $\tilde{E}_{q} \rightarrow \phi_{q}^{*} R_{q}$ is surjective, this shows that the part listed in (1) is also part of $Q_{q}$. Combined, this proves the claim.

We now prove Lemma 3.5. 
Proof of Lemma 3.5. We first show that the contribution of $T_{0}$ in Claim 4.6 is

$$
\int_{s_{\tilde{E}_{q}, \bar{\sigma}_{q}}\left(\left[T_{0}\right]\right)} 1=(-1)^{l}\left[(h-2) 2^{2 h-3}-\sum_{j=0}^{h-2}\left(\begin{array}{c}
2 h+2 \\
h-2-j
\end{array}\right) a_{j}\right] .
$$

We know that $T_{0}$ is a subbundle of $\left.\tilde{E}\right|_{V_{0}}$; its normal bundle in $\left.\tilde{E}\right|_{V_{0}}$ is the pullback of the $R_{q}$, which is the locally free part of $R^{1} \bar{\pi}_{*} \bar{f}^{*} L$. Therefore, the contribution is

$$
(-1)^{l} c_{1}\left(R_{q}\right)\left[Z_{q}\right]
$$

Here $l=h^{0}(L)$ is the dimension of the fiber of $V_{0} \rightarrow Z_{q}$; the sign $(-1)^{l}$ is due to the reason similar to the proof of Proposition 1.1.

Let $(\bar{\pi}, \bar{f}): \overline{\mathcal{C}} \rightarrow Z_{q} \times D$ be the restrictions of $(\pi, f)$ to $Z_{q}$. The degree of $R_{q}$ is the difference of $c_{1}\left(-\bar{\pi}_{!} \bar{f}^{*} L\right)$ and the total degree of the torsion part of $R^{1} \bar{\pi}_{*} \bar{f}^{*} L$. By (4.12), the latter at a point in $\Lambda_{r}^{\prime} \subset \Lambda^{\prime}$ is (4.13) with $\alpha_{i}=2 i-1$; thus the total degree of the torsion part lying over $\Lambda^{\prime}$ is

$$
\sum_{r=0}^{\left[\frac{h-2}{2}\right]} \frac{1}{2} \cdot a_{2 r} \cdot\left(\begin{array}{c}
2 h+2 \\
h-2-2 r
\end{array}\right), \quad a_{2 r}=1+3+5+\cdots+(2 r+1) .
$$

Here the factor $\frac{1}{2}$ is from the trivial $\mathbb{Z}_{2}$ action. Similarly, the degree of the torsion part lying over $\Lambda^{\prime \prime}$ is

$$
\sum_{r=0}^{\left[\frac{h-3}{2}\right]} \frac{1}{2} \cdot a_{2 r+1} \cdot\left(\begin{array}{c}
2 h+2 \\
h-3-2 r
\end{array}\right), \quad a_{2 r+1}=2+4+6+\cdots+(2 r+2) .
$$

Hence, by Proposition 1.1, the contribution from $T_{0}$ is as claimed.

For $u \in \Lambda^{\prime} \cap Z_{q}$, following the proof of Theorem 1.1,

$$
\sum_{i=0}^{r} m_{u, i} \int_{s_{\tilde{E}_{q}, \bar{\sigma} q}^{!}\left(\left[T_{u, i}\right]\right)} 1=\sum_{i=0}^{r}(-1)^{l+r+1-i}(2 i+1)=: b_{2 r} \cdot \frac{(-1)^{l}}{2} \frac{1}{2} .
$$

Here, the sign is from Theorem $1.1 ;(2 i+1)$ is the multiplicity; $1 / 2$ is from the trivial $\mathbb{Z}_{2}$ action.

By the same reason, for $u \in \Lambda^{\prime \prime} \cap Z_{q}$,

$$
\sum_{i=0}^{r} m_{u, i} \int_{s_{\tilde{E}_{q}, \bar{\sigma}_{q}}\left(\left[T_{u, i}\right]\right)} 1=\sum_{i=0}^{r}(-1)^{l+r+1-i}(2 i+2)=: b_{2 r+1} \cdot \frac{(-1)^{l}}{2} \frac{1}{2} .
$$


Combining the above, the contribution to the localized GW invariant of the component $M_{0}$ of branched double covers is

$$
(-1)^{h^{0}(L)}\left[(h-2) 2^{2 h-3}-\sum_{j=0}^{h-2}\left(\begin{array}{c}
2 h+2 \\
h-2-j
\end{array}\right) \cdot \frac{a_{j}-b_{j}}{2}\right] .
$$

Now it is an elementary combinatorial exercise to check that this coincides with the desired $(-1)^{h^{0}(L)}\left(-2^{h-2}\right)$. This completes our proof of Lemma 3.5.

\section{REFERENCES}

1. E. Arbarello, M. Cornalba, P. Griffiths and J. Harris, Geometry of algebraic curves. SpringerVerlag, 1985.

2. K. Behrend and B. Fantechi, The intrinsic normal cone. Invent. Math. 128 (1997), no. 1, 45-88.

3. C. Faber and R. Pandharipande, Hodge integrals and Gromov-Witten theory. Invent. Math. 139 (2000), no. 1, 173-199.

4. J. Harris, Theta characteristics on algebraic curves. Trans. Amer. Math. Soc. 271, no. 2 (1982) 611-638.

5. Y-H. Kiem and J. Li, Gromov-Witten invariants of varieties with holomorphic 2-forms. ArXiv:0707.2986 .

6. Y-H. Kiem and J. Li, Localizing virtual cycles by cosections. ArXiv:1010.0068.

7. Y-H. Kiem and J. Li, Low degree GW invariants of surfaces II. Preprint.

8. J. Lee. Holomorphic 2-forms and Vanishing Theorems for Gromov-Witten Invariants. Canad. Math. Bull. 52 (2009), 87-94.

9. J. Lee and T. Parker. A Structure Theorem for the Gromov-Witten Invariants of Kahler Surfaces. Jour. Diff. Geom. 77 (2007), no.3, 483-513.

10. J. Li and G. Tian. Virtual moduli cycles and Gromov-Witten invariants of algebraic varieties. J. Amer. Math. Soc. 11 (1998), no. 1, 119-174.

11. D. Maulik and R. Pandharipande. New calculations in Gromov-Witten theory. PAMQ 4 (2008), 469-500.

12. A. Okounkov and R. Pandharipande. Virasoro constraints for target curves. Invent. Math. 163 (2006), no. 1, 47-108.

13. C. Radoux. Nombres de Catalan généralisés. Bulletin of Belg. Math. Soc. 4 (1997), no. 2, 289-292.

\section{Young-Hoon Kiem}

Department of Mathematics and Research Institute of Mathematics, Seoul National University, Seoul 151-747, Korea

E-mail: kiem@math.snu.ac.kr 
Jun Li

Department of Mathematics,

Stanford University,

Stanford, USA

E-mail: jli@math.stanford.edu 